\title{
OBSERVABILITY ANALYSIS AND STATE ESTIMATION APPROACH OF POWER SYSTEM
}

\author{
A. M. Agwa ${ }^{1}$ and N. E. Ghanem ${ }^{2}$ \\ ${ }^{1}$ Faculty of Engineering, Al-Azhar University \\ ${ }^{2}$ Ministry of Electricity and Energy
}

\section{ABSTRACT}

This paper introduces a new approach to solve the problem of power system state estimation and check the observability of the measurement scheme. The state estimation is a powerful technique for system monitoring and control. Through a numerical method for observability analysis in power system state estimation based on weighted least square (WLS), the system state will be implemented and the measurement scheme will be judged. In addition to that, investigation for the effects of the measurements redundancy on the accuracy of the estimated variables is also discussed. The technique is applied to IEEE 30 bus system. Test results show the effectiveness of the proposed method to reach the best of the state estimator solution.

\section{Keywords: State Estimation, Weighted Least Square (WLS), Observability Analysis.}

\section{INTRODUCTION}

The electric power grid is a complex interconnected system that may be subjected to blackouts and external disasters like hurricanes. It is necessary for utilities to repair and restore their power system as quickly as possible during extreme conditions. State estimation helps to get a better picture of the power system with an available set of measurements. State Estimation (SE) is defined as a statistical procedure for deriving, from a set of system measurements, a "best" estimate of the system state [1]. In the field of power systems, the objective is to provide a reliable and consistent data base for security monitoring, contingency analysis and system control. To meet the above objectives, SE is required to:

- $\quad$ Produce a "best" estimation of the bus voltage magnitudes and angles.

- $\quad$ Detect, identify and suppress gross measurement errors.

- $\quad$ Produce an estimate of non-metered or lost data points.

The observability problem in power system state estimation was introduced by F. Schweppe and Wildes in [2]. The simplest definition of an observable system is the one that all state variables (bus voltage magnitudes and angles) can be obtained, (calculated, estimated) from the given measurements set. In real-time operation it may happen that the system be formed by observable islands and also by non-observable parts, due to the temporary loss of measurements[3].Analysis of network observability is one of the main functions that needs to be executed in a power system state estimator. The state estimator cannot be executed unless the observable islands are identified ahead of time. The problem of network observability has been studied thoroughly by various researchers in the past. There have been essentially two different approaches taken in solving this problem. The first approach uses a topological model of the power system and its associated measurement set $[2,4,5]$. This approach is fast due to the lack of floating point 
operations. It classifies the measurements as critical or redundant, determines the observable islands, and also identifies the residual spread components. However, it uses the decoupled measurement model where it is assumed that the real and reactive measurements appear in pairs and that there are no ampere measurements (even if they exist, they will be ignored during the observability analysis Extension of the topological observability method to address multiplicity of solutions in the presence of ampere measurements has not yet been reported in the literature. The second approach is based on numerical methods, either using the information (or gain) matrix [6,7], or the measurement Jacobean [8] as the model reflecting the configuration of the system and the measurement set. This approach is rather easy to implement due to its use of already built and factorized matrices of the state estimation problem, and well established sparse factorization and repeat solution routines. While the method is presented for decoupled measurement model [9], extension to the full coupled model is possible. The method can identify observable islands, however classification of the measurements and identification of residual spread components require building the residual sensitivity matrix. Both methods have been implemented and used by various state estimators and they have proved to be efficient and effective alternatives. Recently, it has been demonstrated that uniqueness of the state estimate may be questionable when line current magnitude (ampere) measurements are used in extending the observable islands in power system $[9,10]$. Integration of ampere measurements into the measurement system prohibits the use of decoupled measurement models both for state estimation and for observability analysis. Also, several definitions related to the classification of measurements, such as critical, redundant, etc. should be revisited to account for uniquely and no uniquely observable cases $[11,12]$.A system is said to be non-uniquely observable, if more than one solution can be found for the state estimate based on the given set of measurements. Those branches, whose flows can assume more than one value that satisfies all the system measurements, will be labeled as non-uniquely observable branches. On the other hand, uniquely observable systems will have a unique state estimate and a unique set of branch flows through every system branch. It has been observed that certain conditions on the measurement criticality and measurement residual spread components should be satisfied to guarantee unique observability [11-14]. It was also shown that identification of unobservable and non-uniquely observable branches could be carried out by formulating the problem in newly defined branch variables. The redundancy factor is defined as the ratio of number of measurements to number of state variables in most practical cases. This paper investigates the effects of the redundancy on the accuracy of the estimated variables. If some measurements are added to the state estimation, the state estimation accuracy will be affected. In addition to the reconciliation of our previous results in form of a generalized network observability analysis method, this paper's contributions are a numerical method of classifying measurements, and a non-iterative numerical method of determining non-uniquely observable and unobservable branches. The presented observability method is numerical and it allows determination of network observability, unobservable and, critical measurements, redundancy factor, and residual spread components all within the same framework.

\section{Weighted Least Squares Approach}

The development of the notion of state estimation may be processed along several lines depending on the statistical criterion selected. Of the many criteria that have been examined and used in various applications, WLS criterion is the most commonly encountered method. WLS estimate of our unknown parameter is always expressed as that value of the parameter that gives the minimum of the sum of the squares of the difference between each measured value $\mathrm{Z}$ and the estimated measurements $\hat{Z}$ (the true value being measured, expressed as a function of our unknown parameter) with each squared difference divided or "weighted" by the variance of the meter error [15]. This technique is the weighted least squares based on the successive elimination, which relies on the calculations of the normalized or weighted residuals performed after two to four iterations of the WLS estimation. 
A measurement vector $\underline{Z}$ may be created which contains $m$ measurements from the power system. Measurements include real and reactive power line flows and bus injections, voltage magnitudes at buses, tap ratios for transformers, and more recently, phase angle measurements. Those used in this paper are power flows and injections and voltage magnitudes. The $2 \mathrm{~N}-1$ state variables constitute the state vector $\underline{X}$, which may be related to the measurements $\underline{Z}$ as following:

$\mathrm{Z}^{\text {meas }}=\mathrm{Z}^{\text {true }}+\mathrm{e} \quad$ or,

$\mathrm{Z}=\mathrm{h}(\mathrm{X})+\mathrm{e}$

By deciding upon a criterion for calculating the state estimates $\hat{X}$ from which the estimated measurements and estimated errors are to be computed. It is preferable to minimize the direct sum of the squares of the errors. However, to ensure that the measurements from meters of known greater accuracy are treated more favorably than less accurate measurements, each term in the sum of squares is divided by an appropriate weighting factor $\sigma^{2}$ (the variance of the measurement) to give the objective function.

$$
J(\underline{X})=\left(\sum_{i=1}^{m} \frac{\left[\underline{Z}_{i}^{\text {meas }}-\underline{\hat{Z}}_{\mathrm{i}}\right]^{2}}{\sigma_{\mathrm{i}}{ }^{2}}\right)
$$

The best estimates of the state variables are the estimates that cause the objective function $J(\underline{X})$ to take on its minimum value, which can be formed by the expression:

$$
\begin{aligned}
& \min _{x} J(\underline{X})=\min _{x}\left(\sum_{i=1}^{m} \frac{\left[\underline{Z}_{i}^{\text {meas }}-\underline{\hat{Z}}_{i}\right]^{2}}{\sigma_{i}^{2}}\right) \quad \text { or, } \\
& \min _{x} J(\underline{X})=\min _{x}\left(\sum_{i=1}^{m} \frac{\left[\underline{Z}_{i}^{\text {meas }}-h_{i}(\underline{X})\right]^{2}}{\sigma_{i}{ }^{2}}\right)
\end{aligned}
$$

\section{MEASUREMENTS OBSERVABILITY ANALYSIS}

Consider the measurements Jacobean given by $H$, Jacobean matrix and it's equal to $\frac{\partial \mathrm{h}\left(\mathrm{X}_{\mathrm{i}}\right)}{\partial \mathrm{X}_{\mathrm{i}}}$. Using row pivoting and applying Peters-Wilkinson decomposition $[16,17], H$ can be decomposed into its factors as:

$$
H=L . R
$$

Where:

$H$ : is $\mathrm{m} \times n$ rectangular Jacobean

$L$ : is $\boldsymbol{m} \times n$ lower trapezoidal factor

$\mathrm{m}$ : is the total number of measurement and loop equations

$n$ : is the total number of branch variables

Provided the system is fully observable, this factorization can be carried out numerically by proper row and column pivoting. Otherwise, zero pivots may be encountered during the factorization process at those columns corresponding to some of the existing unobservable branches. Anytime such a zero pivot is encountered, it will be replaced by a value of 1.0 and the corresponding column number will be recorded. The resulting decomposition will appear as below:

$$
\mathrm{H}=\left[\begin{array}{c}
H_{1} \\
H_{2}
\end{array}\right]=\left[\begin{array}{c}
L_{1} \\
\ddot{M}
\end{array}\right]^{[R]}
$$

Where: 
$L_{l}$ is a $n \times n$ lower triangular matrix, $R$ is a $n \times n$ upper triangular matrix, $\mathrm{H}_{2}$ is a $(\boldsymbol{m}-n) \times n$ rectangular matrix, $\mathrm{M}$ is a $(\boldsymbol{m}-n) \times n$ rectangular matrix.

Following the network observability theory described in $[18,19]$, a set of zero measurements should yield zero branch variables for a fully observable system. If a zero pivot is encountered during the factorization of $H$, and it is replaced by $1 . O$, then the measurement in the corresponding row will be assigned an arbitrary but nonzero value. This is equivalent to adding a critical pseudo measurement for the corresponding branch variable. The remaining unobservable branches, those in addition to the already identified ones through the zero pivot columns, can now be determined by carrying out back substitutions for $X$ in the following equation:

$$
R-X=Y
$$

Where $Y_{i}=\left\{\begin{array}{lr}i \text { if } i-\text { th pivot was zero } \\ 0\end{array}\right.$

After back substitution:

$$
X_{j}=\left\{\begin{aligned}
0 & \text { if } j-\text { th branch is observable } \\
\neq 0 & \text { if } j-\text { t } \text { branch is unobservable }
\end{aligned}\right.
$$

If there were no current magnitude (ampere) measurements, observability analysis would have ended here. However, in the presence of ampere measurements, those branches that are identified as observable in the above analysis, may not all be uniquely observable? This means that, there is a chance that more than one state estimation solution, which satisfies all the available measurements, can be reached for the given measurement configuration. Hence, the conventional observability analysis should be extended to determine if such multiple solutions are likely. If they are found to be likely, then those branches, whose flows can assume multiple solutions for the same measurement set, will have to be identified and labelled as non-uniquely observable branches.

The redundancy factor is defined as the ratio of number of measurements to number of state variables in most practical cases.

The paper also investigates the effects of the redundancy on the accuracy of the estimated variables. If some measurements are added to the state estimation, the state estimation accuracy will be affected.

The network is observable because only one pseudo measurement is equal zero during the triangular factorization

$\mathrm{U}=\left[\begin{array}{ccc}1 & \cdots & \cdots \\ \cdots & 1 & \cdots \\ . & . . & 0\end{array}\right]$

If the result of this technique shows multi pseudo measurements are equal to zero during the triangular factorization, it means the system is un-observable

$$
\mathrm{U}=\left[\begin{array}{ccc}
1 & \cdots & \cdots \\
\cdots & 0 & \cdots \\
. & \cdots & 0
\end{array}\right]
$$

\section{CASES STUDY}

In this study, two cases will be introduced here based on the proposed technique of the WLS estimator and observability analysis. The system used in the study is the IEEE 30-bus and 41branch system. The first case uses 75 number of measurements which represent the unobservable system, the second case is the same system of measurements with additional 4 measurements to change it to observable system of measurements. 


\section{Case One}

The measurements placement used for this case is shown in figure (1), which is summarized as follow:

Voltage measurements $=1$

Injection measurements (active and reactive) $=12 \times 2=24$

Power flow measurements (active and reactive) $=25 \times 2=50$

Total number of measurements $(\mathrm{m})=75$

The global redundancy $(\eta)=\mathrm{m} / \mathrm{n}=75 / 59=1.27$

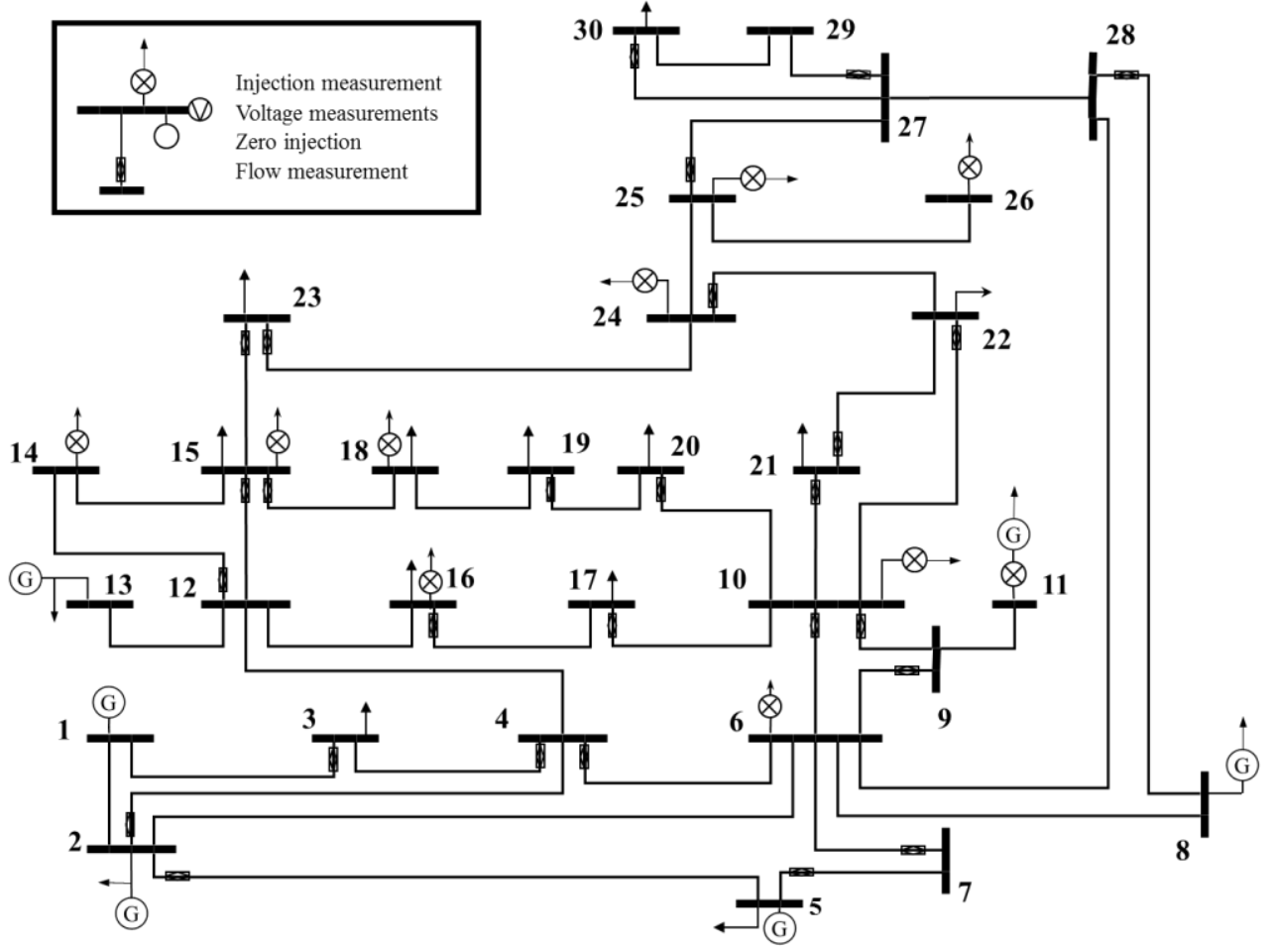

Figure 1: IEEE 30 nodes network with measurements of case one

Running the Matlab program on the proposed method to estimate the system state and check the observability of the measurements configuration, it results 4 zerospivot in the $\mathrm{U}$ matrixin the factorization of the Jacobean matrix.

The result is the system un-observabel systemand cannot continue for state estimationprocedure.

Case two

The measurements placement used for this case is shown in figure (2), which are the measurements of case one adding voltage measurements at buses 11 and 13 and power flow measurements at line 6-8. It can be summarized as follow:

Voltage measurements $=3$

Injection measurements (active and reactive) $=12 \times 2=24$

Power flow measurements (active and reactive) $=26 \times 2=52$

Total number of measurements $(\mathrm{m})=79$

The global redundancy $(\eta)=\mathrm{m} / \mathrm{n}=79 / 59=1.34$ 


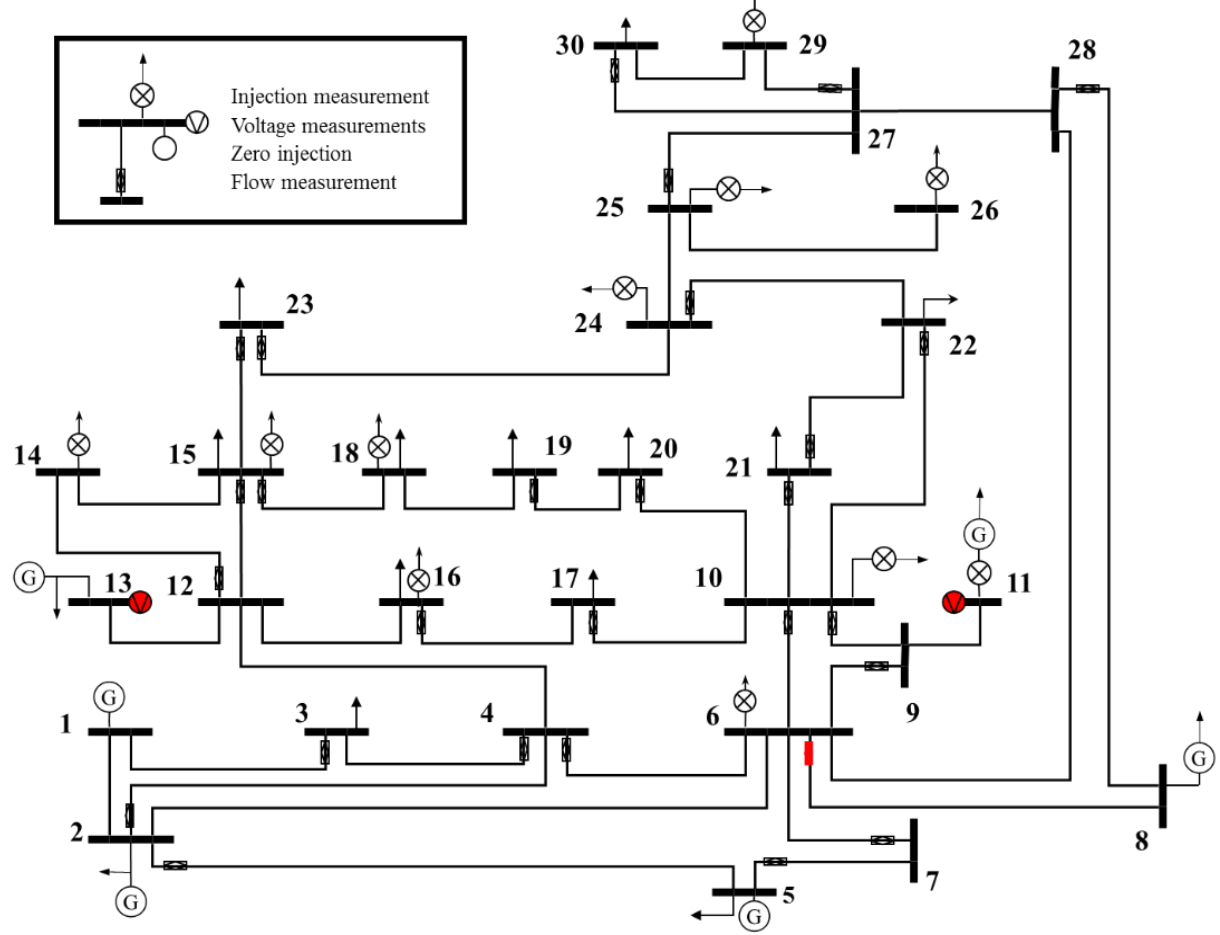

Figure 2: IEEE 30 nodes network with measurements of case two

Running the Matlab program on the proposed method to estimate the system state and check the observability of the measurements configuration, it results non-zero pivot in the $U$ matrix in the factorization of the Jacobean matrix.

The result is the system observabel and the program continue for state estimation procedure. The results of the state estimation program are as shown in Table 1

Table 1: State Estimation results of case study 2

\begin{tabular}{|c|c|c||c|c|c|}
\hline \multicolumn{3}{|c|}{ Voltages in p.u. } & \multicolumn{3}{|c|}{ Angles in deg. } \\
\hline $\mathbf{J}$ & Estimated & Actual & $\mathbf{J}$ & Estimated & Actual \\
\hline $\mathbf{1}$ & 1.0594 & 1.0600 & $\mathbf{1}$ & 0.0000 & 0.0000 \\
\hline $\mathbf{2}$ & 1.0401 & 1.0330 & $\mathbf{2}$ & -5.3672 & -5.2195 \\
\hline $\mathbf{3}$ & 1.0163 & 1.0135 & $\mathbf{3}$ & -7.5430 & -7.5125 \\
\hline $\mathbf{4}$ & 1.0058 & 1.0028 & $\mathbf{4}$ & -9.2928 & -9.2687 \\
\hline $\mathbf{5}$ & 1.0046 & 1.0000 & $\mathbf{5}$ & -14.2210 & -14.2114 \\
\hline $\mathbf{6}$ & 1.0033 & 1.0005 & $\mathbf{6}$ & -11.0566 & -11.0433 \\
\hline $\mathbf{7}$ & 0.9957 & 0.9924 & $\mathbf{7}$ & -12.8910 & -12.8815 \\
\hline $\mathbf{8}$ & 1.0025 & 1.0000 & $\mathbf{8}$ & -11.8002 & -11.8040 \\
\hline $\mathbf{9}$ & 1.0332 & 1.0302 & $\mathbf{9}$ & -14.0810 & -14.0671 \\
\hline $\mathbf{1 0}$ & 1.0165 & 1.0131 & $\mathbf{1 0}$ & -15.6956 & -15.6814 \\
\hline $\mathbf{1 1}$ & 1.0744 & 1.0720 & $\mathbf{1 1}$ & -14.0810 & -14.0671 \\
\hline $\mathbf{1 2}$ & 1.0479 & 1.0455 & $\mathbf{1 2}$ & -15.1939 & -15.2355 \\
\hline
\end{tabular}




\begin{tabular}{|c|c|c||c|c|c|}
\hline \multicolumn{3}{|c|}{ Voltages in p.u. } & \multicolumn{3}{c|}{ Angles in deg. } \\
\hline $\mathbf{J}$ & Estimated & Actual & J & Estimated & Actual \\
\hline $\mathbf{1 3}$ & 1.0738 & 1.0710 & $\mathbf{1 3}$ & -15.1939 & -15.2355 \\
\hline $\mathbf{1 4}$ & 1.0296 & 1.0276 & $\mathbf{1 4}$ & -16.0733 & -16.1303 \\
\hline $\mathbf{1 5}$ & 1.0222 & 1.0203 & $\mathbf{1 5}$ & -16.0634 & -16.1420 \\
\hline $\mathbf{1 6}$ & 1.0273 & 1.0245 & $\mathbf{1 6}$ & -15.6794 & -15.6945 \\
\hline $\mathbf{1 7}$ & 1.0143 & 1.0111 & $\mathbf{1 7}$ & -15.9028 & -15.8994 \\
\hline $\mathbf{1 8}$ & 1.0078 & 1.0053 & $\mathbf{1 8}$ & -16.6572 & -16.7061 \\
\hline $\mathbf{1 9}$ & 1.0025 & 0.9997 & $\mathbf{1 9}$ & -16.8157 & -16.8427 \\
\hline $\mathbf{2 0}$ & 1.0052 & 1.0022 & $\mathbf{2 0}$ & -16.5943 & -16.6109 \\
\hline $\mathbf{2 1}$ & 1.0036 & 1.0006 & $\mathbf{2 1}$ & -16.2551 & -16.1406 \\
\hline $\mathbf{2 2}$ & 1.0069 & 1.0013 & $\mathbf{2 2}$ & -16.0110 & -16.1217 \\
\hline $\mathbf{2 3}$ & 1.0041 & 1.0039 & $\mathbf{2 3}$ & -16.2665 & -16.4357 \\
\hline $\mathbf{2 4}$ & 0.9940 & 0.9906 & $\mathbf{2 4}$ & -16.3327 & -16.4691 \\
\hline $\mathbf{2 5}$ & 0.9972 & 0.9942 & $\mathbf{2 5}$ & -16.0907 & -16.1959 \\
\hline $\mathbf{2 6}$ & 0.9792 & 0.9761 & $\mathbf{2 6}$ & -16.5088 & -16.6356 \\
\hline $\mathbf{2 7}$ & 1.0081 & 1.0053 & $\mathbf{2 7}$ & -15.6716 & -15.7481 \\
\hline $\mathbf{2 8}$ & 0.9994 & 0.9985 & $\mathbf{2 8}$ & -11.7034 & -11.7133 \\
\hline $\mathbf{2 9}$ & 0.9880 & 0.9850 & $\mathbf{2 9}$ & -16.9325 & -17.0233 \\
\hline $\mathbf{3 0}$ & 0.9764 & 0.9733 & $\mathbf{3 0}$ & -17.8416 & -17.9398 \\
\hline
\end{tabular}

\section{CONCLUSION}

This dissertation is concerned to provide a new approach for robust state estimation of power system using WLS.The dissertation also proposed technique to check the observability of the network and determine whether the set of measurements are sufficient in number and location to make state estimation possible or not.The result of state variable in the cases study show the effect of redundancy in state estimation and show that The IEEE 30-bus grid attains improved estimation accuracy due to its increased redundancy ratio. The proposed technique was applied to the IEEE 30 buses test network, and the tabulated results achieved its reliability.

\section{REFERENCES}

[1]. S. A. Nagy, "Bad-Data Detection, Identification And Correction In Electric Power Systems", M.Sc. thesis, Al-Azhar Univ., 2002.

[2]. F. C. Schweppe and J. Wildes, "Power system static state estimation,part I: Exact model," IEEE Trans. Power App. Syst., vol. PAS-89, no.1, pp. 120-125, Jan. 1970.

[3]. K. A. Clements, G. R. Krumpholz, and P. W. Davis, "Power system state estimation with measurement deficiency: An observability/measurement placement algorithm," IEEE Trans. Power App. Syst., vol. PAS-102, no. 7, pp. 2012-2020, Jul. 1983.

[4]. A. Abur and A. G. Exposito, Power System State Estimation- Theory and Implementation", CRC Publisher, 2004. 
[5]. F. C. Schweppe and D. B. Rom, "Power System Static-State Estimation, Part II: Approximate Model",IEEE Transactions on Power Apparatus and Systems, vol. PAS-89, pp. 125-130, 1970.

[6]. F. C. Schweppe, "Power System Static-State Estimation, Part III: Implementation", IEEE Transactions on Power Apparatus and Systems, vol. PAS-89, pp. 130-135, 1970.

[7]. A. G. Phadke, "Synchronized Phasor Measurements - A Historical Overview," Transmission and Distribution Conference and Exhibition 2002: Asia Pacific. IEEE/PES, vol. 1, pp. 476-479, 2002.

[8]. D. Falcb and M. Arias, "State Estimation and Observability Analysis Based on Echelon Forms of the Linearized Measurement Models", IEEE Trans. On Power Systems, Vol. 9(2), May 1994, pp. 979-987.

[9]. A. Abur and A.G. Expbsito, "Detecting Multiple Solutions in State Estimation in the Presence of Current Magnitude Measurements",IEEWPES Winter Meeting, Jan.2125,1996, Baltimore, Paper No: 300- 4-PWRS.

[10]. A. Abur and A.G. Exp6sito, "Multiple Solutions and Unique Observability in State Estimation", Proceedings of the 12th Power Systems Computation Conference, Aug.1923, 1996, Dresden, Germany, pp.1200-1206.

[11]. A. Abur and A.G. Exphito, "Bad Data Identification When Using Ampere Measurements", IEEE/PES Summer Meeting, July 28- Aug.1, 1996, Denver, Colorado, Paper No: 513-2-PWRS.

[12]. S. A. Nagy, Y. Rihan, I. S. Ibrahim, A. S. Adail "Observability Analysis and multi Bad Data Processing Using Phasor Measurements Units in Power System State Estimation”, Journal of Al Azhar University Engineering Sector, Volume 9, Number 31, April 2014.

[13]. S. Nagy, Y. Rihan, I. Salama, A. Adail, "Robust Bad Data Detection and Identification When Using Phasor Measurements in state estimation in power system", Coimbatore Institute of Information Technology (CIIT), Volume 5, Number 12, 2013.

[14]. S. A. Nagy, "Branch Status Topology Errors Detection in power System State Estimation", New York Science Journal, Vol. 5, No. 3, 2012.

[15]. J.J. Grainger, W.D. Stevenson, "Elements of Power System Analysis", McGraw-Hill, 1994.

[16]. G. Peters and J.H. Wilkinson, "The Least Squares Problem and Pseudo-Inverses", The Computer Journal, Vo1.13, No.4, pp.309-316, August 1970.

[17]. J.W. Gu, K.A. Clements, G.R. Krumpholz and P. W. Davis, "The Solution of 111Conditioned Power System State Estimation Problems Via the Method of Petersand Wilkinson" Proceedings of the Power Industry Computer Applications Conference, 1983, pp.239-246

[18]. A. Monticelli and F.F. Wu, "Network Observability: Identification of Observable Islands and Measurement Placement", IEEE Transactions on PAS, Vol.PAS-104, No.5, May 1985, pp.1035-1041.

[19]. A. Monticelli and F.F. Wu, "Network Observability: Theory", IEEE Transactions on PAS, Vol.PAS-104, No.5, May 1985, pp.1042-1048 